\title{
A Randomized Trial of Virtual Reality-Based Cue Exposure Second-Level Therapy and Cognitive Behavior Second-Level Therapy for Bulimia Nervosa and Binge-Eating Disorder: Outcome at Six-Month Followup
}

\author{
Marta Ferrer-Garcia,, Joana Pla- Sanjuanelo,' Antonios Dakanalis, ${ }^{2,3}$ Ferran Vilalta-Abella, \\ Giuseppe Riva, ${ }^{3-10}$ Fernando Fernandez-Aranda, Laura Forcano, ${ }^{6}$ Nadine Riesco, \\ Isabel Sánchez, ${ }^{6}$ Massimo Clerici, Joan Ribas-Sabaté,, Alexis Andreu-Gracia, \\ Neli Escandón-Nagel, Osane Gomez-Tricio,' Virginia Tena, and José Gutiérrez-Maldonado ${ }^{1}$
}

\section{AU4 Abstract}

This article reviews the 6-month followup data of a randomized, multicenter, parallel-group study conducted at five clinical sites in three European cities, which compared two second-level treatments for bulimia nervosa (BN) and binge eating disorder (BED): virtual reality-based cue exposure therapy (VR-CET) versus additional cognitive behavioral therapy (A-CBT). Post-treatment outcomes of this study were already published in FerrerGarcia et al. (2017) and details of its design can be found at clinicaltrials.gov (identifier: NCT02237300, https:// clinicaltrials.gov). This article focuses on the evolution of symptoms assessed after 6 months of followup in a subgroup of 58 patients from the original study. In this study (Ferrer-Garcia et al., 2017) 64 patients with eating disorders (EDs) (35 with BN and 29 with BED), who still showed active episodes of binge eating by the end of a structured CBT program (first-level treatment), were randomly assigned to one of two second-level treatments (A-CBT or VR-CET). Frequency of binge and purge episodes, and attitudinal features of binge-related EDs (bulimia, drive for thinness, and body dissatisfaction) were assessed before starting the second-level treatment $(n=64)$, at the end $(n=64)$, and at 6-month followup $(n=58)$. Mixed between-within subject analyses of variance were used to compare outcomes of both second-level treatments over time. Although both treatment conditions showed statistically significant improvements at the end and after 6-month followup, obtained reductions were greater after VR-CET, regarding binge and purge episodes, as well as the decrease of selfreported tendency to engage in overeating episodes. Accordingly, abstinence from binge episodes were higher in VR-CET than A-CBT at followup (70 percent vs. 26 percent, respectively; $\chi^{2}=11.711, p=0.001$ ). These results provide further support for the use of VR-CET as an effective second-level intervention for BN and BED treatment-resistant patients.

Keywords: cue exposure, virtual reality, bulimia nervosa, binge eating disorder, treatment, followup

${ }^{1}$ Department of Clinical Psychology and Psychobiology, University of Barcelona, Barcelona, Spain.

${ }^{2}$ Department of Medicine and Surgery, University of Milano Bicocca, Milan, Italy.

${ }^{3}$ Department of Brain and Behavioral Sciences, University of Pavia, Pavia, Italy.

${ }^{4}$ Applied Technology for Neuro-Psychology Lab, Istituto Auxologico Italiano, Milan, Italy.

${ }^{5}$ Department of Psychiatry and Mental Health, Igualada General Hospital, Barcelona, Spain.

${ }^{6}$ Department of Psychiatry, University Hospital of Bellvitge-IDIBELL and CIBER Fisiopatología Obesidad y Nutrición (CIBERobn), ISCIII, Barcelona, Spain.

${ }^{7}$ Department of Psychiatry, University Hospital Joan XXIII, Tarragona, Spain.

${ }^{8}$ Centro ABB Tarragona, Tarragona, Spain.

AU3 ${ }^{9}$ Universidad Católica de Temuco, Temuco, Chile.

${ }^{10}$ Department of Psychology, University Cattolica del Sacro Cuore, Milan, Italy. 


\section{Introduction}

AU5 Considerable EVIDEnCE From reviews and metaanalyses of clinical trials supports cognitive behavioral therapy $(\mathrm{CBT})$ as the first-choice treatment for both bulimia nervosa (BN) and binge eating disorder (BED). ${ }^{1-4}$ Overall, outcomes obtained after CBT point to significant improvements in terms of behavioral and psychological features of these disorders. ${ }^{5}$ However, a high percentage of BN and BED patients do not improve after a first-level intervention (i.e. $\mathrm{CBT})^{6,7}$ and the short- and long-term rates of remission from eating disorder (ED) cognitions and behaviors range from around 37 to 69 percent across trials. ${ }^{8}$ Given the high prevalence $^{9}$ and the considerable long-term morbidity of these disorders, the development of new efficacious treatment options is a research priority. ${ }^{8,10}$

Therefore, when the first-level treatment (i.e., CBT) fails or when symptoms do not remit completely, two main approaches have been proposed: either extending CBT with additional sessions ${ }^{11}$ or conducting second-level treatments ${ }^{12,13}$ targeting specific features related to the patient's history, their clinical or psychopathological features, and/or their response to previous treatments. Several techniques have been proposed to target specific features associated with poor response, such as cognitive dysfunction, ${ }^{14}$ emotional dysregulation, ${ }^{15,16}$ or the urge to binge in response to food-related cues $^{17}$ (i.e., food craving). In this regard, cue exposure therapy (CET), aims to extinguish/habituate craving and anxiety responses to food-related cues, ${ }^{18,19}$ and thus reduce the associated risk of overeating. Several studies, including case reports, ${ }^{20-24}$ and nonrandomized ${ }^{25}$ and randomized ${ }^{26,27}$ controlled studies, have reported the efficacy of CET in reducing food craving and anxiety, and the positive results in terms of reducing episodes of binge eating and associated purging behaviors. However, several logistical constraints of CET (e.g., in vivo exposure requires patients to bring sufficient quantities of binge foods to the therapy sessions, and it is usually limited to specific/proximal cues), have led to the use of virtual reality (VR).

VR technology shows several advantages over the traditional exposure procedures ${ }^{28}$ of in vivo or imagery exposure. Although in vivo exposure, when possible, is usually the best option, it sometimes presents major problems, such as patients' refusal to participate, difficulties in maintaining the necessary levels of confidentiality and safety (when exposure is conducted in the real situation), the time taken to travel to the exposure situation, and low control. These limitations can be partially overcome when in vivo exposure is conducted in the clinician's office; however, this option only allows exposure to proximal cues (e.g., foods), not to contextual cues (e.g., kitchen). As for imagery exposure, it resolves some of drawbacks mentioned above, but, in turn, requires a significant cognitive effort and causes considerable fatigue; these drawbacks increase the risk that patients will use avoidance strategies, as clinicians cannot fully control the scenario that patients are imagining. VR technology, on the other hand, allows us to develop virtual simulations of everyday life scenarios, where exposure to food-related stimuli is conducted in more controlled and ecological conditions. Compared with in vivo exposure, VR provides a greater degree of confidentiality and safety, allows the inclusion of both contextual and proximal cues, and prevents unforeseen events during exposure; it also helps to adapt exposure to the needs of each patient, thus reducing any resistance to treatment and increasing motivation. Compared with imagery exposure, VR stimulates several sensory modalities (e.g., auditory and visual), facilitating the involvement of participants who have trouble imagining scenes, and helps therapists identify the stimuli causing a specific emotional response, as they know what the patient is seeing at each particular moment.

VR-based cue exposure therapy ${ }^{29}$ (VR-CET) has been reported to reduce food cravings and anxiety, ${ }^{30,31}$ as well as eliminate episodes of binge eating ${ }^{31,32}$ after systematic exposure to virtual food-related contexts and cues. There is also evidence of the ability of food-related VR-based environments to elicit anxiety and craving responses similar to those expected in real life in both healthy and clinical (i.e., patient with $\mathrm{BN}$ and BED) groups. ${ }^{33-40}$

As previously described, ${ }^{32}$ a randomized controlled trial was conducted to assess the efficacy of VR-CET as a secondlevel treatment for BN and BED patients. The study showed that both second-level treatments (VR-CBT vs. additional cognitive behavior therapy [A-CBT]) improved all dimensional measures of outcome (i.e., clinician-rated frequency of episodes of binge eating and purging, self-reported tendency to engage in episodes of uncontrollable overeating, drive for thinness (DT), body dissatisfaction [BD], anxiety, and food craving), but a better overall short-term outcome (i.e., at post-treatment) was observed in the VR-CET group, ${ }^{32}$ with a significantly higher reduction in number of binge and purge episodes and self-reported tendency to engage in episodes of overeating, food craving, and anxiety than the A-CBT group. Furthermore, binge-purging abstinence rates were also significantly higher in VR-CET. ${ }^{32}$ This study addressed whether these positive outcomes were maintained in the long term (i.e., at 6-month followup) in a subgroup of 58 patients who participated in the original study.

Specifically, we aimed to assess whether outcomes at the 6-month followup of the two second-level treatments (VRCET vs. A-CBT) were maintained and to determine whether binge/purge abstinence rates in the VR-CET group (who received an intervention focused on the reduction of anxiety and craving associated with food-related stimuli) were lower than the binge/purge abstinence rates in the A-CBT group.

\section{Methods}

\section{Participants and procedure}

In this study, we analyze and discuss the 6-month followup outcomes of the abovementioned randomized, multicenter parallel-group study ${ }^{32}$ that was conducted at five clinical sites in three European cities (Barcelona and Tarragona, Spain, and Milan, Italy) between February 2015 and March 2016. The study protocol was approved by the ethics review board of each local institution (clinical site) and of the University of Barcelona, and written informed consent was obtained from all participants after the procedure was fully explained. Details about the design of the study can be found at clinicaltrials.gov (identifier: NCT02237300, https:// clinicaltrials.gov) and in a previous publication, ${ }^{32}$ where results of both second-level treatments are summarized. Table 1 summarizes the main characteristics of the two $4 \mathrm{~T} 1$ second-level treatments (A-CBT and VR-CET) and Table $2<\mathrm{T} 2$ 
VIRTUAL REALITY CUE EXPOSURE THERAPY FOR BN AND BED

Table 1. Second-Level Treatments' Main Characteristics

Second-level treatments

Cognitive behavioral treatment (A-CBT)

Six twice-weekly individual 60-minute sessions held over 3 weeks.

Sessions focused on reinforcing the strategies and skills developed during the first-level CBT intervention (e.g., psychoeducation; meal planning; identifying and challenging overvalued cognitions about food, eating, weight, and shape; identifying cues for bulimic behaviors and use of strategies such as stimulus control to manage cues; relapse prevention) and assessing in greater depth aspects of the disorder that remained problematic after finishing the first-level intervention (e.g., body image disturbance; food craving; and dysfunctional cognitions), as suggested by Eldredge et al. ${ }^{11}$
Virtual reality-based cue exposure therapy (VR-CET)

Six twice-weekly individual 60-minute (maximum) sessions held over 3 weeks.

Sessions focused on diminishing or extinguishing the conditioned psychophysiological reactivity (i.e., anxiety and craving) to food-related cues, which have been associated with binge eating behavior. ${ }^{19-22}$

Based on the theoretical approaches of Jansen ${ }^{19}$ and Martínez-Mallén et al., ${ }^{22}$ participants were exposed to a hierarchy of virtual reality scenarios simulating different food-related situations where they were exposed to the foods that they had previously rated as the ones that produced the highest levels of craving from a list of 30 items. ${ }^{36,41}$

During exposure, the patient was able to handle the virtual foods using the laptop's mouse, but could not eat them (exposure with response prevention).

Exposure to each hierarchy step ended when the participant's anxiety ${ }^{40}$ level (assessed on a visual analog scale from 0 to 100 displayed on the laptop's monitor) decreased by 40 percent in relation to the level registered at the start of the exposure session or after 60 minutes of exposure.

Note: A more detailed description of the VR-CET procedure has been previously published. ${ }^{31,32}$

A-CBT, additional cognitive behavioral therapy; CBT, cognitive behavioral therapy.

includes details about VR system used to administrate the VR-CET.

The initial sample consisted of 35 patients with $\mathrm{BN}$ and 29 with BED, who showed active episodes of binge eating during the last 2 weeks of a structured program of CBT (firstlevel treatment), and who agreed to participate in the study. The presence of current comorbid severe mental disorders (substance use disorders, bipolar disorder, and psychosis) was considered an exclusion criterion, but not the use of antidepressant medication. ${ }^{42-44}$ Participants were randomly assigned to one of two second-level treatments (A-CBT or VR-CET) using Biased Coin Randomization developed by Efron, ${ }^{32}$ and were assessed in the prerandomization phase (i.e., at the end of treatment with the structured CBT program), at the end of the second-level treatments, and at a 6month followup session. All randomized participants $(N=64)$ finished the second-level treatments and completed the post-treatment assessment. ${ }^{32}$ However, only 58 patients completed the 6-month followup assessment (9.4 percent dropout rate). It was not possible to reestablish contact with four participants and, among those who agreed to attend the followup session, two did not attend the appointment and did not wish to arrange another one. Thus, in the final sample, there were 27 patients in the A-CBT group (15.6 percent dropout rate) and 31 patients in the VR-CET group (3.1 percent dropout rate).

Table 2. Virtual Reality System's Main Components

\section{Hardware}

5.6-in 3D laptop (i.e., three-dimensional view of the VR scenarios) with polarized glasses.

Laptop's mouse, to move around the virtual environment and to interact with the food placed inside (i.e., the food can be lifted, rotated, and zoomed, but not eaten).

Earphones, to isolate participants from the real world.

\section{Software}

The software comprises a library with four contexts (kitchen, dining room, bedroom, and bakery-café) and 30 foods (e.g., ice cream, pizza, cookies, and popcorn).

Before initiating CET, users are exposed to bidimensional images of the 30 foods and the four contexts, and food craving elicited per item is assessed using a visual analogue scale (from 0 to 100).

Based on this information, the software creates an individualized exposure hierarchy with 3D interactive environments and foods (Figs. 1 and 2).

Note: Given that previous research ${ }^{30}$ showed that prolonged exposure to food-related virtual environments decreased the craving initially reported in a nonclinical sample both when using a head mounted display and when using a 3D laptop, the second option was chosen so as to reduce the technological complexity and the risk of simulator sickness ${ }^{28}$ (i.e., nausea, disorientation, headache, sweating, dizziness, general fatigue, eye strain, and blurred vision).

3D, three-dimensional; CET, cue exposure therapy. 


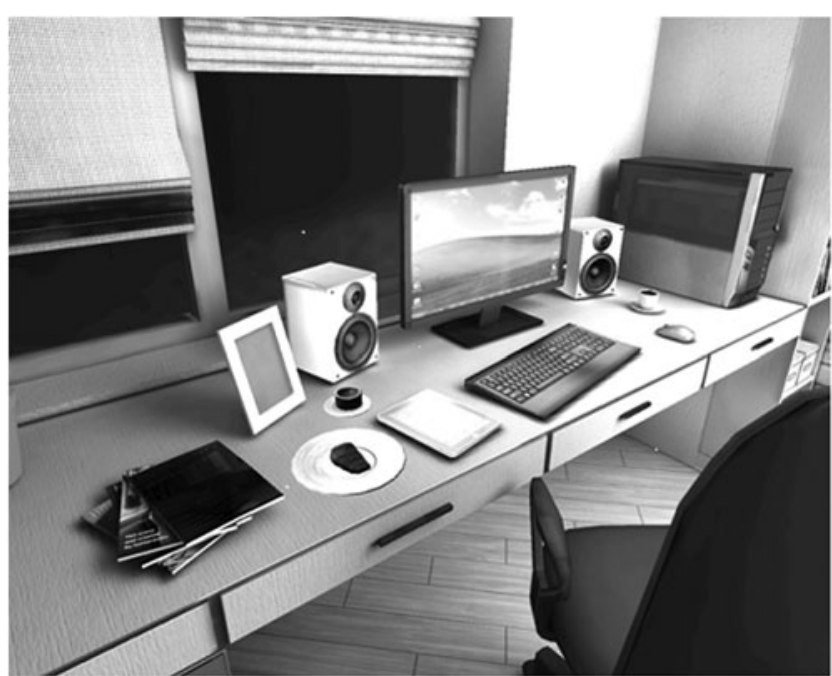

FIG. 1. Picture of the virtual bedroom.

Table 3 contains descriptive data on these groups. There were no significant differences between the groups in the prerandomization phase in terms of gender $\left(\chi^{2}=0.037\right.$, $p=0.781)$ or diagnosis $\left(\chi^{2}=0.318, p=0.573\right)$. The A-CBT group consisted of eight men (29.6 percent) and 19 women (70.4 percent) and the VR-CET group consisted of 11 men (35.5 percent) and 20 women (64.5 percent). As regard to diagnosis, the A-CBT group included 16 patients with BN (59.3 percent) and 11 patients with $\operatorname{BED}$ (40.7 percent), and the VR-CET included 15 patients with $\mathrm{BN}$ (48.4 percent) and 16 patients with BED (51.6 percent). There were no significant differences found in the prerandomization phase in terms of age, body mass index, duration of the ED, clinicianrated frequency of episodes of binge eating and purging, selfreported tendency to engage in episodes of uncontrollable overeating (i.e., bulimia subscale on the EDI-3), selfreported measures of attitudinal features of EDs (i.e., DT and BD subscales on the EDI-3), anxiety, or food craving $(p>0.05)$.

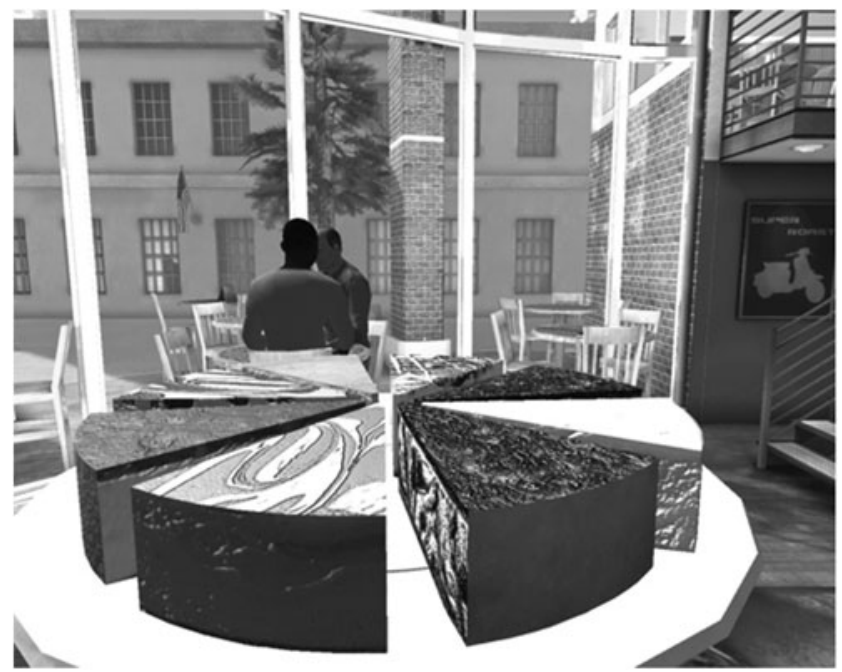

FIG. 2. Picture of the virtual bakery-café.

\section{Measures}

The ED Examination-Interview ${ }^{45} 12.0 \mathrm{D}$ adapted to cover the specified time frame, that is, past 2 weeks, ${ }^{12}$ was used to assess the core behavioral features of $\mathrm{BN}$ and BED, specifically the frequency of binge eating episodes and purging (i.e., self-induced vomiting and laxative and diuretic use, in the case of patients with $\mathrm{BN}$ ). Attitudinal features of $\mathrm{BN}$ and BED were assessed using the eight-item bulimia subscale (B), the seven-item DT, and the 10-item BD scales of the ED Inventory- $3^{46}$; all EDI- 3 scales ( $\alpha$ 's $\geq 0.87$ ) are rated on a five point scale (ranging from 0 to 4 ). Finally, the State-Trait Anxiety Inventory ${ }^{47}$ (STAI) ${ }^{47}$ and the Food Craving Questionnaire $^{48}$ Trait (FCQ-T) and State (FCQ-S) were also included to assess specific variables targeted by VR-CET. ${ }^{30}$ The STAI consists of two 20-item scales (rated on a fourpoint scale from 1 to 4 ) for measuring the level of anxiety as a state (STAI-S; i.e., at the time of evaluation) and trait (STAI-T) ( $\alpha$ 's $\geq 0.89$ ). The FCQ-S (consisting of 15 items rated on a five-point scale, ranging from 1 to 5) and the FCQ$\mathrm{T}$ (consisting of 39 items rated on a six-point scale, ranging from 1 to 6) were designed to assess state (i.e., at the time of evaluation) and trait food craving ( $\alpha$ 's $\geq 0.92)$.

\section{Statistical analyses}

Mixed between-within subject analyses of variance were used to compare the outcomes of both second-level treatments over time, with the treatment group (VR-CET vs. A-CBT) being the between-subject factor and the time (prerandomization/pretest, end-of treatment/post-test, and 6month followup) being the within-subject factor. Frequency of binge eating and purge episodes (rated by the clinicians), self-reported tendency to engage in episodes of uncontrollable overeating (assessed using the EDI-3 bulimia scale), and attitudinal ED features (assessed using the EDI-3 DT and BD scales) were the outcomes included in the analyses. In those analyses in which the sphericity assumption was violated (Mauchly's test $p<0.05$ ), degrees of freedom were corrected using Greenhouse-Geisser estimates of sphericity (ع). Although the analyses in this study were consistent with those carried out in our earlier research, ${ }^{31}$ the interpretation of results is focused on effect sizes and confidence intervals (CIs) around these effects. Since the traditional null hypothesis significance testing shows important limitations, the use of effect sizes and CIs has previously been proposed as a more accurate interpretation approach. ${ }^{49-51}$ Accordingly, Cohen's $d$ was used to assess the effect of pairwise comparisons found when combining the outcomes of both ACBT and VR-CET interventions across the three assessment sessions (pretreatment, post-treatment, and 6-month followup). Interpretation of effect sizes was based on Cohen ${ }^{52}$ for Cohen's $d$, considering values $<0.20$ as an indicator of nonexistence of effect, values between 0.21 and 0.49 as an indicator of a small effect, values between 0.50 and 0.70 as an indicator of moderate effect, and values equal to or $>0.80$ as an indicator of large effects; and on eta squared $\left(\eta^{2}\right)$ values, considering 0.01 a small effect size, 0.06 a moderate effect size, and 0.14 a large effect size. Finally, $\chi^{2}$ tests were conducted to compare abstinence from the pathological behavior (i.e., no episodes of binge eating and purging) at the end of the second-level treatment and at 6-month followup in both groups. Given that, by definition, BED patients do not 
Table 3. Outcome Measures in A-CBT and VR-Cet Groups at Pretest, Post-Test and Six-Month Followup

\begin{tabular}{|c|c|c|c|c|c|c|}
\hline & \multicolumn{2}{|c|}{ Pretest measures } & \multicolumn{2}{|c|}{ Post-test measures } & \multicolumn{2}{|c|}{ 6-Month followup } \\
\hline & $\begin{array}{c}A-C B T \\
\mathrm{n}=27\end{array}$ & $\begin{array}{c}V R-C E T \\
\mathrm{n}=31\end{array}$ & $\begin{array}{c}A-C B T \\
\mathrm{n}=27\end{array}$ & $\begin{array}{c}V R-C E T \\
\mathrm{n}=31\end{array}$ & $\begin{array}{c}A-C B T \\
\mathrm{n}=27\end{array}$ & $\begin{array}{c}V R-C E T \\
\mathrm{n}=31\end{array}$ \\
\hline Age & $34.37(9.55)$ & $34.64(10.16)$ & - & - & 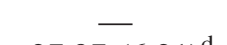 & - \\
\hline BMI & $28.69(7.15)$ & $27.27(4.88)$ & - & - & $27.97(6.21)^{\mathrm{d}}$ & $26.36(4.66)$ \\
\hline ED duration & $13.23(11.73)^{\mathrm{c}}$ & $12.39(8.60)$ & - & - & - & - \\
\hline Binges $^{\mathrm{a}}$ & $12.85(6.99)$ & $11.13(6.36)$ & $6.33(5.80)$ & $0.77(1.02)$ & $6.33(6.30)$ & $1.19(2.83)$ \\
\hline Purges $^{b}$ & $11.69(7.45)$ & $10.33(7.76)$ & $5.06(6.03)$ & $0.47(0.83)$ & $6.31(7.06)$ & $1.53(3.70)$ \\
\hline EDI-DT & $17.92(7.32)$ & $17.61(7.43)$ & 13.55 (7.28) & $14.87(5.98)$ & $11.81(6.61)$ & $11.29(6.26)$ \\
\hline EDI_B & $20.63(6.93)$ & $20.06(7.53)$ & 17.92 (6.79) & $6.42(5.47)$ & $14.81(7.94)$ & $4.90(5.97)$ \\
\hline EDI-BD & $25.59(9.56)$ & $24.48(8.28)$ & $20.00(9.53)$ & $19.52(5.95)$ & $18.37(9.00)$ & $16.81(8.47)$ \\
\hline FCQ-T & 151.27 (32.70) & $149.35(3.28)$ & $138.67(35.92)$ & $84.19(47.83)$ & $123.41(35.05)$ & $76.90(46.38)$ \\
\hline FCQ-S & $41.96(14.44)$ & $43.19(15.16)$ & 36.37 (14.88) & $23.45(10.84)$ & 36.78 (15.77) & $23.32(11.01)$ \\
\hline STAII-T & $38.96(10.18)$ & $37.38(13.01)$ & $35.30(9.51)$ & $26.64(8.98)$ & $31.48(8.89)$ & $24.10(10.80)$ \\
\hline STAI-S & 33.63 (12.95) & $33.13(18.11)$ & $30.74(11.50)$ & $21.10(8.30)$ & $25.81(10.92)$ & $19.81(9.54)$ \\
\hline
\end{tabular}

Note: Only participants who completed the assessment at followup are included.

${ }^{a}$ Number of binge episodes during the last 2 weeks

${ }^{b}$ Number of purge episodes during the last 2 weeks (only BN patients, $n=16$ in A-CBT group and $n=15$ in VR-CET group).

${ }^{\mathrm{c}} n=26$.

$\mathrm{d}_{n=24}$.

ED duration, eating disorder duration in years; BMI, body mass index; EDI, eating disorders inventory; DT, drive for thinness; B, bulimia; BD, body dissatisfaction; BN, bulimia nervosa; STAI, State-Trait Anxiety Inventory; FCQ, Food craving questionnaire; T, trait; $\mathrm{S}$, state.

engage in purging behaviors, ${ }^{44}$ all the analyses conducted with this variable only included BN patients. Analyses were conducted using SPSS Statistics for Windows version 23.

\section{Results}

Patients in both second-level treatment groups (A-CBT and VR-CET) showed a significant reduction in symptomatology post-treatment that was maintained at 6-month followup. However, overall improvement was greater in the VR-CET group than in the A-CBT group. This was particularly evident in the rates of abstinence from binging and purging. At the 6-month followup, the percentage of binge abstinence increased (from 53 percent at the post-treatment) ${ }^{32}$ to 70 percent in the VR-CET group (22 of the 31 patients), while in the A-CBT group, it was maintained (25.9 percent, 7 of the 27 patients) $\left(\chi^{2}=11.711, p=0.001\right)$. Among BN patients, the percentage of participants who achieved abstinence from purging episodes at followup was also higher in the VRCET group (11 of the 15 patients, 73.3 percent) than in the ACBT group ( 5 of the 16 patients, 31.3 percent) $\left(\chi^{2}=5.490\right.$, $p=0.019)$. Consistent with these results, the VR-CET group showed a lower self-reported tendency to engage in episodes of uncontrollable overeating (assessed using the bulimia scale of the EDI-3) than the A-CBT group at the end of the secondlevel treatment and, again, at the 6-month followup, even when no differences were found among groups pretreatment. Most importantly, differences between the A-CBT and VRCET groups post-treatment and at followup showed very large effect sizes for binge episodes $(d=1.381$ and $d=1.078$, respectively), purge episodes ( $d=1.051$ and $d=0.840$, respectively), and the bulimia scale of the EDI-3 ( $d=1.888$ and AU6 T5 $d=1.426$, respectively), as shown in Table 5 .

T4 Table 4 summarizes the results of mixed between-within subject analyses of variance. The effect of time (pretreatment, post-treatment, and 6-month followup) was large for all measured variables ( $\eta^{2}$ ranging from 0.291 to 0.619 ), meaning that all patients showed a significant reduction in behavioral (binge and purge episodes) and attitudinal (DT, bulimia, and BD assessed using the EDI-3) features of ED, as well as food craving and anxiety, across assessment sessions. On the other hand, the interaction between time and group showed moderate to very large effects only on frequency of

Table 4. Mixed Between-Within Subject Analyses of Variance Comparing Treatment Groups on Behavioral (Number of Binge Eating and Purge Episodes) and Attitudinal Eating Disorder Features (Bulimia, Drive for Thinness, and Body Dissatisfaction), and Cue ExposureRelated Variables (Food Craving and AnXiety) at Pretreatment, at Post-Treatment, AND AT Six-Month Followup

\begin{tabular}{|c|c|c|c|c|c|c|}
\hline & \multicolumn{3}{|c|}{$\begin{array}{l}\text { Time (pretest-post- } \\
\text { test-followup) }\end{array}$} & \multicolumn{3}{|c|}{ Time $\times$ group } \\
\hline & $\mathrm{F}_{(2,55)}$ & $\mathrm{p}$ & $\eta^{2}$ & $\mathrm{~F}_{(2,55)}$ & $\mathrm{p}$ & $\eta^{2}$ \\
\hline Binges $^{\mathrm{a}}$ & 90.931 & $<0.001$ & 0.619 & 4.348 & 0.023 & 0.072 \\
\hline Purges ${ }^{b}$ & 38.856 & $<0.001$ & 0.573 & 1.809 & 0.183 & 0.059 \\
\hline EDI-DT & 49.862 & $<0.001$ & 0.471 & 1.299 & 0.274 & 0.023 \\
\hline EDI-B & 62.532 & $<0.001$ & 0.528 & 17.988 & $<0.001$ & 0.243 \\
\hline EDI-BD & 41.462 & $<0.001$ & 0.425 & 0.208 & 0.786 & 0.004 \\
\hline FCQ-T & 74.913 & $<0.001$ & 0.572 & 21.665 & $<0.001$ & 0.279 \\
\hline FCQ-S & 42.991 & $<0.001$ & 0.434 & 14.091 & $<0.001$ & 0.201 \\
\hline STAI-T & 41.634 & $<0.001$ & 0.426 & 5.232 & 0.013 & 0.085 \\
\hline STAI-S & 22.957 & $<0.001$ & 0.291 & 4.121 & 0.032 & 0.069 \\
\hline
\end{tabular}

Note: Only participants who completed the assessment at followup are included.

${ }^{\mathrm{a}}$ Number of binge episodes during the last 2 weeks.

${ }^{\mathrm{b}}$ Number of purge episodes during the last 2 weeks (only BN patients, $n=16$ in A-CBT group and $n=15$ in VR-CET-group; $d f=29$ ). 


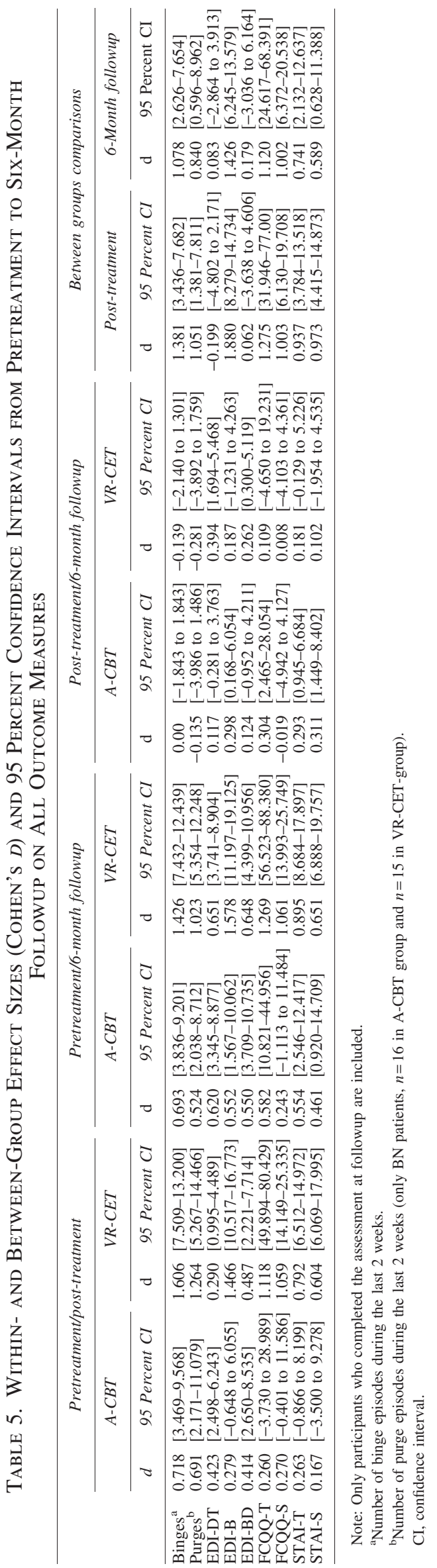

binge and purge episodes, bulimia scale of the EDI-3, trait/ state food craving, and trait/state anxiety reported by participants. On the whole, the reduction of scores in these variables was significantly higher in the VR-CET group than in the A-CBT group at post-treatment, despite the fact that no difference between groups had been found at pretreatment. These improvements were maintained at 6-month followup without great changes in either group. Table 5 provides more detailed information about the differences in outcomes over time and between treatment groups. As mentioned above, all participants showed a significant reduction in symptom severity (based on self-reported frequency of binge and purge episodes and questionnaires) post-treatment. However, effect sizes in the A-CBT group ranged from small to moderate, while effect sizes in the VR-CET group ranged from moderate to very large (with one exception: Cohen's $d$ for EDIDT was 0.290 , a small effect). When comparing measures pretreatment and at 6-month followup, the effect sizes in the VR-CET group were even larger, ranging from 0.648 (moderate effect) to 1.578 (very large effect). Nevertheless, comparisons between the outcome post-treatment and at 6month followup showed nonsignificant small or very small size effects in both the A-CBT and VR-CET groups, providing support for the continued maintenance of improvements achieved post-treatment after 6 months.

Finally, between-group comparisons (Table 5) revealed that the frequency of binge and purge episodes $(d=1.078$, and $d=0.840$, respectively), self-reported tendency to engage in overeating episodes (assessed with the bulimia scale of the EDI-3; $d=1.426$ ), food craving (assessed as a trait and as a state; $d=1.120$, and $d=1.002$, respectively), and anxiety (assessed as a trait and as a state; $d=0.741$, and $d=0.589$, respectively) at 6-month followup were significantly lower in patients in the VR-CET group than in patients of the ACBT group, as also found post-treatment, with most of the effect sizes of these differences being large or very large.

\section{Discussion}

Followup data collected in this study showed that outcomes after two second-level treatments (A-CBT or VR-CET) applied to patients with $\mathrm{BN}$ and $\mathrm{BED}$ resistant to standard treatment (i.e., structured CBT intervention) were maintained for at least 6 months. Most importantly, abstinence from binge episodes increased to 70 percent in the VR-CET group. Recovery rates (understood as the elimination of binge behavior) used to be low (from 11 percent to 26 percent) in treatmentresistant patients with BN and BED. ${ }^{53}$ Previous research found that the addition of CET sessions, as a second-level treatment, to a CBT-based intervention produced a binging abstinence rate (assessed for the last year) of 54 percent at 5year followup. ${ }^{17}$ So, rates around 20 percent in the A-CBT group and around 50 percent in the VR-CET group would be expected. Results obtained at the end of second-level treatment $^{32}$ were consistent with the abovementioned data (53 percent in the VR-CET group and 25 percent in the A-CBT group). However, the fact that the percentage abstinence from binging increased even more during followup in the VR-CET group provides support for those models that associate food craving and anxiety with binge behavior, and that predict that lower levels of food craving and food-related anxiety reduce the risk of binge behavior. ${ }^{19,22}$ 
VR-CET is focused on reducing craving and anxiety responses in patients when they are exposed to food-related cues (e.g., pizza) and contexts (e.g., bakery), with the aim of reducing the risk of engaging in binge behaviors (and, consequently, the risk of purging). Consistent with this objective, self-reported food craving (assessed using the FCQ-T/S) and anxiety (assessed using the STAI-T/S) in this group were greatly reduced post-treatment and at the 6-month followup, compared with pretreatment scores (with very large effects for FCQ scores and moderate-to-large effects for STAI). Furthermore, both post-treatment and at 6-month followup, levels of food craving and anxiety reported by the A-CBT group were much higher than in the VR-CET group (with very large effects for FCQ scores and moderateto-large effects for STAI). Differences in food craving and anxiety may explain the superiority of VR-CET in the maintenance and even improvement of binge episode abstinence rates at followup. Coherent with abstinence from binging, the rate of abstinence for purge episodes in BN patients was higher in the VR-CET group (73.3 percent vs. 31.5 percent). As purge episodes are aimed at counteracting the caloric intake and weight gain from binge eating, ${ }^{54,55}$ a reduction in binge episodes would be expected to lead to a reduction in purge episodes.

As previously reported, ${ }^{32}$ all patients in both second-level treatments showed a significant reduction in self-reported tendency to engage in uncontrollable overeating episodes, DT, and BD (assessed using the B, DT. and BD scales of EDI-3) post-treatment. However, scores on the DT and BD scales still fitted the clinical range (EDI-DT scores between 9 and 22 and EDI-BD scores between 13 and 28, according to Elosua et al. ${ }^{56}$ ) in both groups. Thus, we hypothesized that normalization of eating patterns may improve attitudinal features of EDs, such as DT and BD, in the long term. ${ }^{57-60}$ The 6-month followup data did not support this statement. Even though DT and BD scores were lower at the 6-month followup than post-treatment, the effects of the differences were small and scores remained in the clinical range (although near the lower end of the range). On the other hand, the bulimia scale scores in VR-CET patients were almost in the range of nonclinical significance $(\leq 4$, according to Elosua et al. ${ }^{56}$ ) at the 6-month followup, whereas the scores of participants in the A-CBT group still fell within the clinical range. The EDI-3 bulimia scale assesses the tendency to engage in episodes of uncontrollable overeating. As mentioned above, the main objective of VR-CET is to reduce cue-elicited food craving and anxiety responses to prevent binge behavior, so our results were consistent with the efficacy of intervention.

The limitations of the randomized controlled trial were summarized in a previous publication, ${ }^{32}$ the most relevant being the following: the lack of supervision of the structured CBT first-level intervention at the different participating sites and not having controlled for a possible placebo effect from switching treatment in the VR-CET group. Furthermore, while VR-CET focused on CET, the A-CBT did not include this component. Consequently, an in vivo and/or photograph-based CET condition should be added in future research to assess the benefits of VR over other exposure methods. Despite these drawbacks, our followup data provide added support to the use of VR-CET as an effective second-level intervention for $\mathrm{BN}$ and $\mathrm{BED}$ treatment- resistant patients, as previously suggested. ${ }^{18,22,24}$ Patients in the VR-CET group not only showed a huge improvement after only six VR-CET sessions but also the outcome was maintained and even improved at followup. Although treatment-resistant patients assigned to A-CBT also benefitted from additional CBT sessions, ${ }^{11}$ the results of this study show that the use of VR for targeting specific features associated with poor response, ${ }^{13,42,43,61}$ such as urge to binge in response to a cue ${ }^{26}$ and anxiety experienced simultaneously in the presence of binge-related cues, ${ }^{22,24,26,30,31}$ is a better strategy for treating resistant BN and BED patients. ${ }^{62}$ Consequently, future research should focus on the underlying conceptual model and mechanisms of action of VRCET $^{29,61,62}$ to strengthen its rationale and optimize its clinical application.

\section{Acknowledgments}

This study was supported by the Spanish Ministry of Science and Innovation (MINECO/FEDER/UE; project PSI2011-28801: "Virtual Reality Cue-Exposure Treatment for Bulimia Nervosa") and by a scholarship granted to one of the authors (J.P.-S.) (Grants for the recruitment of earlystage research staff FI-DGR 2014 supported by the Ministry of Economy and Knowledge, Generalitat de Catalunya). A.D., M.C., and G.R. are supported by a grant (PRIN; project 201597WTTM: "Unlocking the memory of the body: Virtual reality in anorexia nervosa") from the Italian Ministry of Education, Universities and Research. F.F.-A. is supported by FIS PI14/290. CIBEROBN is an initiative of ISCIII.

\section{Disclosure Statement}

No competing financial interests exist.

\section{References}

1. Linardon J, Fairburn CG, Fitzsimmons-Craft EE, et al. The empirical status of the third-wave behaviour therapies for the treatment of eating disorders: a systematic review. Clinical Psychology Review 2017; 58:125-140.

2. Linardon J, Wade TD, de la Piedad Garcia X, et al. The efficacy of cognitive-behavioral therapy for eating disorders: a systematic review and meta-analysis. Journal of Consulting and Clinical Psychology 2017; 85:1080-1094.

3. Peat CM, Berkman ND, Lohr KN, et al. Comparative effectiveness of treatments for binge-eating disorder: systematic review and network meta-analysis. European Eating Disorders Review 2017; 25:317-328.

4. Vall E, Wade TD. Predictors of treatment outcome in individuals with eating disorders: a systematic review and meta-analysis. International Journal of Eating Disorders 2015; 48:946-971.

5. Hay PJ, Bacaltchuk J, Stefano S, et al. Psychological treatments for bulimia nervosa and binging. Cochrane Database of Systematic Reviews 2009; 4:1-170.

6. Nazar BP, Gregor LK, Albano G, et al. Early response to treatment in eating disorders: a systematic review and a diagnostic test accuracy metaanalysis. European Eating Disorders Review 2017; 25:67-79.

7. Linardon J, de la Piedad Garcia X, Brennan L. Predictors, moderators, and mediators of treatment outcome following manualised cognitive-behavioural therapy for eating dis- 
orders: a systematic review. European Eating Disorders Review 2017; 25:3-12.

8. Hudson JI, Hiripi E, Pope HG, et al. The prevalence and correlates of eating disorders in the National Comorbidity Survey replication. Biological Psychiatry 2007; 61:348358.

9. Fischer S, Meyer AH, Dremmel D, et al. Short-term cognitive-behavioral therapy for binge eating disorder: long-term efficacy and predictors of long-term treatment success. Behaviour Research and Therapy 2014; 58:36-42.

10. Schaumberg K, Welch E, Breithaupt L, et al. The science behind the academy for eating disorders' nine truths about eating disorders. European Eating Disorders Review 2017; 25:432-450.

11. Eldredge KL, Agras WS, Arnow B, et al. The effects of extending cognitive-behavioral therapy for binge eating disorder among initial treatment nonresponders. International Journal of Eating Disorders 1997; 21:347-352.

12. Mitchell JE, Halmi K, Wilson GT, et al. A randomized secondary treatment study of women with bulimia nervosa who fail to respond to CBT. International Journal of Eating Disorders 2002; 32:271-281.

13. Halmi KA. Perplexities of treatment resistence in eating disorders. BMC Psychiatry 2013; 13:292.

14. Tchanturia K, Giombini L, Leppanen J, et al. Evidence for cognitive remediation therapy in young people with anorexia nervosa: systematic review and meta-analysis of the literature. European Eating Disorders Review 2017; 25: 227-236.

15. Fernandez-Aranda F, Jimenez-Murcia S, Santamaría JJ, et al. The use of videogames as complementary therapeutic tool for cognitive behavioral therapy in bulimia nervosa patients. Cyberpsychology Behavior and Social Networking 2015; 18:744-751.

16. Mallorquí-Bagué N, Vintró-Alcaraz C, Sánchez I, et al. Emotion regulation as a transdiagnostic feature among eating disorders: cross-sectional and longitudinal approach. European Eating Disorders Review 2018; 26:53-61.

17. McIntosh VVW, Carter FA, Bulik CM, et al. Five-year outcome of cognitive behavioral therapy and exposure with response prevention for bulimia nervosa. Psychological Medicine 2011; 41:1061-1071.

18. Koskina A, Campbell IC, Schmidt U. Exposure therapy in eating disorders revisited. Neuroscience and Biobehavioral Reviews 2013; 37:193-208.

19. Jansen A. A learning model of binge eating: cue reactivity and cue exposure. Behaviour Research and Therapy 1998; 36:257-272.

20. Jansen A, Van Den Hout MA, De Loof C, et al. A case of bulimia successfully treated by cue exposure. Journal of Behavior Therapy and Experimental Psychiatry 1989; 20: 327-332.

21. Kennedy SH, Katz R, Neitzert CS, et al. Exposure with response prevention treatment of anorexia nervosa-bulimic subtype and bulimia nervosa. Behavior Research and Therapy 1995; 6:685-689.

22. Martínez-Mallén E, Castro-Fornieles J, Lázaro L, et al. Cue exposure in the treatment of resistant adolescent bulimia nervosa. International Journal of Eating Disorders 2007; 40:596-601.

23. Schmidt U, Marks IM. Exposure plus prevention of bingeing vs. exposure plus prevention of vomiting in bulimia nervosa. A crossover study. Journal of Nervous and Mental Disease 1989; 177:259-266.
24. Toro J, Cervera M, Feliu MH, et al. Cue exposure in the treatment of resistant bulimia nervosa. International Journal of Eating Disorders 2003; 34:1-8.

25. Jansen A, Broekmate J, Heymans M. Cue-exposure vs selfcontrol in the treatment of binge eating: a pilot study. Behaviour Research and Therapy 1992; 30:235-241.

26. Bulik CM, Sullivan PF, Carter FA, et al. The role of exposure with response prevention in the cognitivebehavioural therapy for bulimia nervosa. Psychological Medicine 1998; 28:611-623.

27. Cooper PJ, Steere J. A comparison of two psychological treatments for bulimia nervosa: implications for models of maintenance. Behaviour Research and Therapy 1995; 33: 875-885.

28. Ferrer-Garcia M, Gutiérrez-Maldonado J. The use of virtual reality in the study, assessment, and treatment of body image in eating disorders and nonclinical samples: a review of the literature. Body Image 2012; 9:1-11.

29. Gutiérrez-Maldonado J, Wiederhold BK, Riva G. Future directions: how virtual reality can further improve the assessment and treatment of eating disorders and obesity. Cyberpsychology, Behavior, and Social Networking 2016; 19:148-153.

30. Gutiérrez-Maldonado J, Pla-Sanjuanelo J, Ferrer-Garcia M. Cue-exposure software for the treatment of bulimia nervosa and binge eating disorder. Psicothema 2016; 28:363-369.

31. Pla-Sanjuanelo J, Ferrer-Garcia M, Vilalta-Abella F, et al. Using virtual reality for cue-exposure therapy in a case of bulimia nervosa. Annual Review of CyberTherapy and Telemedicine 2016; 14:155-160.

32. Ferrer-García M, Gutiérrez-Maldonado J, Pla-Sanjuanelo J, et al. A randomised controlled comparison of second-level treatment approaches for treatment-resistant adults with bulimia nervosa and binge eating disorder: assessing the benefits of virtual reality cue exposure therapy. European Eating Disorders Review 2017; 25:479-490.

33. Agliaro-López M, Ferrer-Garcia M, Pla-Sanjuanelo J, et al. Inducción de craving por comida mediante realidad virtual no inmersiva. [Inducing food craving by means of non immersive virtual reality]. Revista de Psicopatología y Psicología Clínica 2014; 19:243-251.

34. Ferrer-Garcia M, Gutiérrez-Maldonado J, Caqueo-Urízar A, et al. The validity of virtual environments for eliciting emotional responses in patients with eating disorders and in controls. Behavior Modification 2009; 33:830-854.

35. Ferrer-Garcia M, Gutiérrez-Maldonado J, Pla-Sanjuanelo J. Cue-elicited anxiety and craving for food using virtual reality scenarios. Studies in Health Technology and Informatics 2013; 191:105-109.

36. Ferrer-Garcia M, Gutiérrez-Maldonado J, Pla-Sanjuanelo J, et al. Development of a VR application for binge eating treatment: identification of contexts and cues related to bingeing behavior in Spanish and Italian patients. Studies in Health Technology and Informatics 2014; 199:71-75.

37. Ferrer-Garcia M, Gutierrez-Maldonado J, Pla-Sanjuanelo J, et al. External eating as a predictor of cue-reactivity to food-related virtual environments. Annual Review of $\mathrm{Cy}$ bertherapy and Telemedicine 2015; 13:117-122.

38. Gorini A, Griez E, Petrova A, et al. Assessment of the emotional responses produced by exposure to real food, virtual food and photographs of food in patients affected by eating disorders. Annals of General Psychiatry 2010; 9:30.

39. Perpiñá C, Roncero M, Fernández-Aranda F, et al. Clinical validation of a virtual environment for normalizing eating 
pattern in eating disorders. Comprehensive Psychiatry 2013; 54:680-686.

40. Pla-Sanjuanelo J, Ferrer-Garcia M, Vilalta-Abella F, et al. Testing virtual reality-based cue-exposure software: which cue-elicited responses best discriminate between patients with eating disorders and healthy controls? Eating and Weight Disorders-Studies on Anorexia, Bulimia and Obesity 2017; [Epub ahead of print]; DOI: 10.1007/s40519017-0419-4.

41. Pla-Sanjuanelo J, Ferrer-Garcia M, Gutiérrez-Maldonado J, et al. Identifying specific cues and contexts related to bingeing behavior for the development of effective virtual environments. Appetite 2017; 87:81-89.

42. Dakanalis A, Bartoli F, Caslini M, et al. Validity and clinical utility of the DSM-5 severity specifier for bulimia nervosa: results from a multisite sample of patients who received evidence-based treatment. European Archives of Psychiatry and Clinical Neuroscience 2016; 267:823-829.

43. Dakanalis A, Colmegna F, Riva G, et al. Validity and utility of the DSM-5 severity specifier for binge eating disorder. International Journal of Eating Disorders 2017; 50:917-923.

44. Fairburn CG, Cooper Z, Doll HA, et al. Transdiagnostic cognitive-behavioural therapy for patients with eating disorders: a two site trial with 60-week follow-up. American Journal of Psychiatry 2009; 166:311-319.

45. Fairburn CG, Cooper Z. (1993) The eating disorder examination (twelfth edition). In Fairburn CG, Wilson GT, eds. Binge eating: nature, assessment and treatment. 12th ed. New York: Guilford Press, pp. 317-360.

46. Garner DM. (2004) EDI 3: eating disorder inventory-3: professional manual. Lutz, FL: Psychological Assessment Resources.

47. Spielberger CD, Gorsuch RL, Lushene R. (1983) Manual for the state-trait anxiety inventory. Palo Alto, CA: Consulting Psychologists Press.

48. Cepeda-Benito A, Gleaves DH, Fernández MC, et al. The development and validation of Spanish versions of the state and trait food cravings questionnaires. Behaviour, Research and Therapy 2000; 38:1125-1138.

49. Cumming G. The new statistics: why and how. Psychological Science 2014; 25:7-29.

50. Faulkner C, Fidler F, Cumming G. The value of RCT evidence depends on the quality of statistical analysis. Behaviour Research and Therapy 2008; 46:270-281.

51. Sullivan G, Feinn R. Using effect size-or why the $p$-value is not enough. Journal of Graduate Medical Education 2012; 4:279-282.
52. Cohen J. (1988). Statistical power analysis for the behavioral sciences. Hillsdale, NJ: Erlbaum.

53. Mitchell JE, Agras S, Crow S, et al. Stepped care and cognitive-behavioural therapy for bulimia nervosa: randomised trial. The British Journal of Psychiatry 2011; 198: 391-397.

54. Al-Adawi S, Bax B, Bryant-Waugh R, et al. Revision of ICD-status update on feeding and eating disorders. Advances in Eating Disorders 2013; 1:10-20.

55. American Psychiatric Association. (2013) Diagnostic and statistical manual of mental disorders. 5th ed. Arlington, VA: American Psychiatric Publishing.

56. Elosua P, López-Jáuregui A, Sánchez-Sánchez F. (2010) Manual técnico con la adaptación al euskera del eating 4 AU9 disorder inventory-3. Madrid, Spain: TEA Ediciones.

57. Grilo CM, Crosby RD, Wilson GT, et al. 12-Month followup of fluoxetine and cognitive behavioral therapy for binge eating disorder. Journal of Consulting and Clinical Psychology 2012; 80:1108-1113.

58. Roncero M, Perpiñá C. Normalizing the eating pattern with virtual reality for bulimia nervosa: a case report. Revista Mexicana de Trastornos Alimentarios 2015; 6:152-159.

59. Sepúlveda A, Carrobles JA, Gandarillas AM. Associated factors of unhealthy eating patterns among Spanish university students by gender. Spanish Journal of Psychology 2010; 13:364-375.

60. Serino S, Dakanalis A, Gaudio S. Out of body, out of space: impaired reference frame processing in eating disorders. Psychiatry Research 2015; 230:732-734.

61. Riva G, Gaudio S. Locked to a wrong body: eating disorders as the outcome of a primary disturbance in multisensory body integration. Consciousness Cognition 2018; 59: 57-59.

62. Riva G. Letter to the Editor: virtual reality in the treatment of eating and weight disorders. Psychological Medicine 2017; 47:2567-2568.

Address correspondence to:

Prof. Marta Ferrer-Garcia

Department of Clinical Psychology and Psychobiology

University of Barcelona

Passeig de la Vall d'Hebron, 171

Barcelona 08035

Spain

E-mail: martaferrerg@ub.edu 


\section{AUTHOR QUERY FOR CYBER-2017-0675-VER9-FERRER-GARCIA_1P}

AU1: Please identify (highlight or circle) all authors' surnames for accurate indexing citations.

AU2: Please mention the authors' degree abbreviations (e.g., MS, MD, and PhD).

AU3: Please provide department name in affiliations 8 and 9.

AU4: Please note no URLs and References are to be cited in the Abstract. Please move the reference to an appropriate place in the text (citing it according to the journal's style) and add it to the reference list, renumbering references depending on the journal's style. Please replace the URL in the Abstract with the source/organization name that applies to the URL.

AU5: The Publisher requests for readability that no paragraph exceeds 15 typeset lines. Please check for long paragraphs and divide where needed.

AU6: Citations of Tables 4 and 5 in nonsequential order. Please check.

AU7: Ref. 61 is a duplicate of ref. 13. Hence, duplicate entry has been deleted and reference citations has been renumbered accordingly. Please confirm the edit.

AU8: References are arranged to maintain sequential order in text. Please check.

AU9: Please provide the title in English for ref. 56.

\section{EDITOR QUERY FOR CYBER-2017-0675-VER9-FERRER-GARCIA_1P}

EQ1: Please confirm the $<\mathrm{MT}>$ of the article. 